Pacific

Journal of

Mathematics

\title{
K-THEORY AND HOMOTOPIES OF 2-COCYCLES ON HIGHER-RANK GRAPHS
}

\section{ELIZABETH GILLASPY}




\title{
K-THEORY AND HOMOTOPIES OF 2-COCYCLES ON HIGHER-RANK GRAPHS
}

\author{
ELIZABETH GILlaSPY
}

\begin{abstract}
This paper continues our investigation into the question of when a homotopy of 2-cocycles on a locally compact Hausdorff groupoid gives rise to an isomorphism of the $K$-theory groups of the twisted groupoid $C^{*}$-algebras. Our main result, which builds on work by Kumjian, Pask, and Sims, shows that a homotopy of 2-cocycles on a row-finite higher-rank graph $\Lambda$ gives rise to twisted groupoid $C^{*}$-algebras with isomorphic $K$-theory groups. (The groupoid in question is the path groupoid of $\Lambda$.) We also establish a technical result: any homotopy of 2-cocycles on a locally compact Hausdorff groupoid $\mathcal{G}$ gives rise to an upper semicontinuous bundle of $C^{*}$-algebras.
\end{abstract}

\section{Introduction}

Higher-rank graphs, or $k$-graphs, provide a $k$-dimensional analogue of directed graphs. They were introduced by Kumjian and Pask [2000] to provide a combinatorial model for the higher-rank Cuntz-Krieger algebras studied by Robertson and Steger [1999]. Much of the interest in the $C^{*}$-algebras $C^{*}(\Lambda)$ associated to $k$-graphs $\Lambda$ stems from the multiple ways one can model $C^{*}(\Lambda)$ - the $k$-graph $\Lambda$ reflects many of the properties of $C^{*}(\Lambda)$, but we can also describe $C^{*}(\Lambda)$ as a universal $C^{*}$-algebra for certain generators and relations, or as a groupoid $C^{*}$-algebra $C^{*}(\Lambda) \cong C^{*}\left(\mathcal{G}_{\Lambda}\right)$.

The class of groupoids includes groups, group actions, equivalence relations, and group bundles. Renault [1980] initiated the study of groupoid $C^{*}$-algebras, and the theory and applications of groupoid $C^{*}$-algebras have since been developed by many researchers. Given a 2-cocycle $\omega \in Z^{2}(\mathcal{G}, \mathbb{T})$ on a groupoid $\mathcal{G}$, Renault also explained how to construct the twisted groupoid $C^{*}$-algebra $C^{*}(\mathcal{G}, \omega)$. These objects have received relatively little attention until quite recently, but it has now become clear that twisted groupoid $C^{*}$-algebras can help answer many questions about the structure of untwisted groupoid $C^{*}$-algebras (see [Muhly and Williams 1992; Muhly et al. 1996; Clark and an Huef 2012; an Huef et al. 2011; Brown

MSC2010: 46L05, 46L80.

Keywords: higher-rank graph, twisted groupoid $C^{*}$-algebra, $K$-theory, twisted $k$-graph $C^{*}$-algebra, upper semicontinuous $C^{*}$-bundle, $C_{0}(X)$-algebra, groupoid, 2-cocycle. 
and an Huef 2014]), as well as classifying those $C^{*}$-algebras which admit diagonal subalgebras (also known as Cartan subalgebras) — see [Kumjian 1986]. In another direction, [Tu et al. 2004] establishes a connection between the $K$-theory of twisted groupoid $C^{*}$-algebras and the classification of $D$-brane charges in string theory.

Two recent papers have explored the effect of a homotopy $\left\{\omega_{t}\right\}_{t \in[0,1]}$ of 2-cocycles on the $K$-theory of the twisted groupoid $C^{*}$-algebras. Echterhoff, Lück, Phillips, and Walters showed in [Echterhoff et al. 2010, Theorem 1.9] that if $G$ is a group that satisfies the Baum-Connes conjecture with respect to the coefficient algebras $\mathcal{K}$ and $C([0,1], \mathcal{K})$, and if $\left\{\omega_{t}\right\}_{t \in[0,1]}$ is a homotopy of 2-cocycles on $G$, then the $K$-theory groups of the reduced twisted group $C^{*}$-algebras are unperturbed by the homotopy

$$
K_{*}\left(C_{r}^{*}\left(G, \omega_{0}\right)\right) \cong K_{*}\left(C_{r}^{*}\left(G, \omega_{1}\right)\right) .
$$

In particular, taking $G=\mathbb{Z}^{2}$, we obtain another proof of the fact, established by Pimsner and Voiculescu [1980], that all of the rotation algebras $\left\{A_{\theta}\right\}_{\theta \in[0,1]}$ have isomorphic $K$-theory groups.

Kumjian, Pask, and Sims also studied the effect of a homotopy of 2-cocycles on $K$-theory in [Kumjian et al. 2013]. Theorem 5.4 of [Kumjian et al. 2013] establishes that if $\Lambda$ is a row-finite, source-free $k$-graph and $c$ is a 2-cocycle on $\Lambda$ such that $c(\lambda, \mu)=e^{2 \pi i \sigma(\lambda, \mu)}$ for some $\mathbb{R}$-valued 2-cocycle $\sigma$, then we have $K_{*}\left(C^{*}(\Lambda)\right) \cong K_{*}\left(C^{*}(\Lambda, c)\right)$. Defining $c_{t}(\lambda, \mu)=e^{2 \pi i t \sigma(\lambda, \mu)}$ for $t \in[0,1]$ gives us a homotopy of 2-cocycles linking $c$ and the trivial 2-cocycle. Moreover, Corollary 7.8 of [Kumjian et al. 2015] tells us that $C^{*}(\Lambda, c)$ is isomorphic to a twisted groupoid $C^{*}$-algebra $C^{*}\left(\mathcal{G}_{\Lambda}, \omega_{c}\right)$. Thus, we can view [Kumjian et al. 2013, Theorem 5.4] as a result about homotopic 2-cocycles on groupoids.

Inspired by the above-mentioned results, we have begun exploring the question of when a homotopy of 2-cocycles on a locally compact Hausdorff groupoid $\mathcal{G}$ induces an isomorphism of the $K$-theory groups of the (full or reduced) twisted groupoid $C^{*}$-algebras. In a previous article [Gillaspy 2015], we extended the abovementioned Theorem 1.9 of [Echterhoff et al. 2010] to the case when $\mathcal{G}=G \ltimes X$ is a transformation group, where $X$ is locally compact Hausdorff and $G$ satisfies the Baum-Connes conjecture with coefficients.

We prove the following generalization of [Kumjian et al. 2013, Theorem 5.4].

Theorem 4.1. Let $\Lambda$ be a row-finite $k$-graph with no sources and let $\left\{c_{t}\right\}_{t \in[0,1]}$ be a homotopy of 2-cocycles in $\underline{Z}^{2}(\Lambda, \mathbb{T})$. Then $\left\{c_{t}\right\}_{t \in[0,1]}$ gives rise to a homotopy $\left\{\sigma_{c_{t}}\right\}_{t \in[0,1]}$ of 2-cocycles on $\mathcal{G}_{\Lambda}$ such that

$$
K_{*}\left(C^{*}\left(\mathcal{G}_{\Lambda}, \sigma_{c_{0}}\right)\right) \cong K_{*}\left(C^{*}\left(\mathcal{G}_{\Lambda}, \sigma_{c_{1}}\right)\right) .
$$

As of this writing, we are unaware of any examples of groupoids $\mathcal{G}$ and homotopies $\omega=\left\{\omega_{t}\right\}_{t \in[0,1]}$ of 2-cocycles on $\mathcal{G}$ where the homotopy does not induce an isomorphism of the $K$-theory groups of the twisted groupoid $C^{*}$-algebras. 
Outline. This paper begins by recalling the definitions of a higher-rank graph and of a groupoid in Section 2, as well as the definition of a 2-cocycle in each category, and sketching the procedure by which we can construct a $C^{*}$-algebra from these objects. In Section 3 we define a homotopy of 2-cocycles on a $k$-graph and on a groupoid, and show that the definitions are compatible. We also prove a technical result (Theorem 3.3), namely, that a homotopy $\left\{\omega_{t}\right\}_{t \in[0,1]}$ of 2-cocycles on a groupoid $\mathcal{G}$ gives rise to a $C([0,1])$-algebra with fiber algebra $C^{*}\left(\mathcal{G}, \omega_{t}\right)$ at $t \in[0,1]$. We expect that this result will prove useful in future work, as we search for more classes of groupoids where a homotopy of 2-cocycles induces an isomorphism of the $K$-theory groups of the twisted groupoid $C^{*}$-algebras.

In Section 4 we begin the proof of Theorem 4.1. Our proof technique consists of proving a stronger version of Theorem 4.1 in a simple case, and then showing how to use this simple case to obtain our desired result for general $k$-graphs. To be precise, Proposition 4.2 shows that when the degree map $d$ on $\Lambda$ satisfies $d(\lambda)=b(s(\lambda))-b(r(\lambda))$ for all $\lambda \in \Lambda$, the $C([0,1])$-algebra associated to a homotopy of 2-cocycles on $\Lambda$ is actually a trivial continuous field. We then show how to exploit the triviality of the continuous field in this special case to see that a homotopy of 2-cocycles on any row-finite, source-free $k$-graph $\Lambda$ induces an isomorphism $K_{*}\left(C^{*}\left(\Lambda, c_{0}\right)\right) \cong K_{*}\left(C^{*}\left(\Lambda, c_{1}\right)\right)$. The argument closely parallels Section 5 of [Kumjian et al. 2013].

\section{Groupoids and $k$-graphs}

Definition 2.1 [Kumjian and Pask 2000, Definition 1.1]. A higher-rank graph of degree $k$, or a $k$-graph, is a nonempty countable small category $\Lambda$ equipped with a functor $d: \Lambda \rightarrow \mathbb{N}^{k}$ (the degree map) satisfying the following factorization property: Given a morphism $\lambda \in \Lambda$ with $d(\lambda)=m+n$, there exist unique $\mu, v \in \Lambda$ such that $\lambda=\mu \nu, d(\mu)=m$, and $d(\nu)=n$.

The simplest example of a $k$-graph is $\mathbb{N}^{k}$, equipped with the identity morphism id : $\mathbb{N}^{k} \rightarrow \mathbb{N}^{k}$.

In this article we use the arrows-only picture of category theory, so that we think of the objects of a category $\Lambda$ as identity morphisms. Hence, $\lambda \in \Lambda$ means that $\lambda$ is a morphism in $\Lambda$. Given an element $\lambda$ in a category $\Lambda$, write $s(\lambda)$ for the domain, or source, of the morphism $\lambda$, and write $r(\lambda)$ for its target, or range. We say a $k$-graph $\Lambda$ is row-finite if, for any $v \in \operatorname{Obj}(\Lambda)$ and any $n \in \mathbb{N}^{k}$, the set

$$
v \Lambda^{n}:=\{\lambda \in \Lambda: r(\lambda)=v, d(\lambda)=n\}
$$

is finite. We say $\Lambda$ has no sources if $v \Lambda^{n} \neq \varnothing$ for every $v \in \operatorname{Obj}(\Lambda)$ and every $n \in \mathbb{N}^{k}$. We only consider $k$-graphs which are row-finite and have no sources, 
since these are the $k$-graphs which we can study via the groupoid method that was introduced in [Kumjian and Pask 2000] and which we will explain in Section 2.

Definition 2.2 [Renault 1980, Definition I.1.12; Kumjian et al. 2015, Section 3]. For a category $\Lambda$, let $\Lambda^{* 2}=\left\{\left(\lambda_{1}, \lambda_{2}\right) \in \Lambda \times \Lambda: s\left(\lambda_{1}\right)=r\left(\lambda_{2}\right)\right\}$. A function $c: \Lambda^{* 2} \rightarrow \mathbb{T}$ is called a 2 -cocycle on $\Lambda$ if

$$
c(\lambda, \mu \nu) c(\mu, v)=c(\lambda \mu, \nu) c(\lambda, \mu)
$$

whenever $(\lambda, \mu),(\mu, v) \in \Lambda^{* 2}$ and $c(\lambda, s(\lambda))=c(r(\lambda), \lambda)=1$ for all $\lambda \in \Lambda$. We write $\underline{Z}^{2}(\Lambda, \mathbb{T})$ for the set of 2-cocycles on $\Lambda$.

If $c, \tilde{c}$ are two 2-cocycles on $\Lambda$, we say that $c, \tilde{c}$ are cohomologous if there exists a function $b: \Lambda \rightarrow \mathbb{T}$ such that

$$
\tilde{c}(\mu, v):=b(\mu) b(v) b(\mu \nu)^{-1} c(\mu, v)=\delta b(\mu, v) c(\mu, v) \quad \text { for all }(\mu, v) \in \Lambda^{* 2} .
$$

We note that cohomologous 2-cocycles give rise to isomorphic twisted $C^{*}$-algebras (see [Kumjian et al. 2015, Proposition 5.6; Renault 1980, Proposition II.1.2]).

The only cocycles we consider in this paper are 2-cocycles, so we will occasionally drop the " 2 " and refer to them simply as cocycles.

Definition 2.3 [Kumjian et al. 2015, Definition 5.2]. The twisted higher-rank-graph algebra $C^{*}(\Lambda, c)$ associated to a $k$-graph $\Lambda$ and a 2-cocycle $c$ on $\Lambda$ is the universal $C^{*}$-algebra generated by a collection $\left\{s_{\lambda}\right\}_{\lambda \in \Lambda}$ of partial isometries satisfying the following twisted Cuntz-Krieger relations:

(CK1) $\left\{s_{v}\right\}_{v \in \mathrm{Obj}(\Lambda)}$ is a collection of mutually orthogonal projections;

(CK2) $s_{\mu} s_{v}=c(\mu, v) s_{\mu \nu}$ whenever $s(\mu)=r(v)$;

(CK3) $s_{\mu}^{*} s_{\mu}=s_{s(\mu)}$ for all $\mu \in \Lambda$;

(CK4) $s_{v}=\sum_{\mu \in v \Lambda^{n}} s_{\mu} s_{\mu}^{*}$ for all $v \in \operatorname{Obj}(\Lambda)$ and all $n \in \mathbb{N}^{k}$.

Note that every $k$-graph $\Lambda$ admits at least one 2-cocycle: the trivial cocycle, obtained by setting $c(\lambda, \mu)=1$ for all $(\lambda, \mu) \in \Lambda^{* 2}$. In this case, the definition above of $C^{*}(\Lambda, c)$ agrees with that of $C^{*}(\Lambda)$ given in [Kumjian and Pask 2000, Definition 1.5]. For example, if $\Lambda$ is $\mathbb{N}^{k}$ and $c$ is the trivial cocycle, then we have $C^{*}(\Lambda, c) \cong C\left(\mathbb{T}^{k}\right)$. More generally, if $\Lambda$ is $\mathbb{N}^{2}$, let $c_{\theta}: \Lambda^{* 2} \rightarrow \mathbb{T}$ be given by $c_{\theta}((m, n),(j, k))=e^{2 \pi i \theta n j}$. Then $c_{\theta}$ is a 2-cocycle on $\Lambda$ and $C^{*}\left(\Lambda, c_{\theta}\right)$ is isomorphic to the rotation algebra $A_{\theta}$.

Groupoids. In this section, we review the construction of a twisted groupoid $C^{*}$-algebra set forth in [Renault 1980], as well as the procedure given in the seminal article [Kumjian and Pask 2000] for associating a groupoid to a $k$-graph. Theorem 3.3 applies to arbitrary locally compact Hausdorff groupoids, so we 
present in full generality all the definitions necessary for the construction of a twisted groupoid $C^{*}$-algebra.

A groupoid $\mathcal{G}$ is a small category with inverses. We use the notation of [Renault 1980] to denote groupoid elements and operations; for example, $\mathcal{G}^{(2)} \subseteq \mathcal{G} \times \mathcal{G}$ denotes the set of composable pairs and $\mathcal{G}^{(0)}$ denotes the unit space. If $u \in \mathcal{G}^{(0)}$, we write

$$
\mathcal{G}_{u}=\{x \in \mathcal{G}: s(x)=u\}, \quad \mathcal{G}^{u}=\{x \in \mathcal{G}: r(x)=u\} .
$$

In this article, we restrict our attention to groupoids which admit a locally compact Hausdorff topology in which the operations of composition (or multiplication) and inversion are continuous.

In addition to the groupoids associated to $k$-graphs, examples of groupoids include groups, vector bundles, and transformation groups. For details and examples, see [Goehle 2009].

Given a row-finite, source-free $k$-graph $\Lambda$, Section 2 of [Kumjian and Pask 2000] describes how to form the associated path groupoid $\mathcal{G}_{\Lambda}$ :

Definition 2.4 [Kumjian and Pask 2000, Example 1.7(ii)]. Define the $k$-graph $\Omega_{k}$ to be the category with $\operatorname{Obj}\left(\Omega_{k}\right)=\mathbb{N}^{k}$ and morphisms $\Omega_{k}=\left\{(m, n) \in \mathbb{N}^{k} \times \mathbb{N}^{k}: n \geq m\right\}$. We have $r(m, n)=m, s(m, n)=n, d(m, n)=n-m$. Composition in $\Omega_{k}$ is given by $(m, n)(n, \ell)=(m, \ell)$.

For a $k$-graph $\Lambda$, let $\Lambda^{\infty}$ denote the set of degree-preserving functors $x: \Omega_{k} \rightarrow \Lambda$. When $k=1$, the elements $x \in \Lambda^{\infty}$ are the infinite paths in $\Lambda$.

Given $p \in \mathbb{N}^{k}$, define $\sigma^{p}: \Lambda^{\infty} \rightarrow \Lambda^{\infty}$ by $\sigma^{p}(x)(m, n)=x(m+p, n+p)$. When $\Lambda$ is row-finite and source-free, Proposition 2.3 in [Kumjian and Pask 2000] shows that if $\lambda \in \Lambda, x \in \Lambda^{\infty}$ satisfy $s(\lambda)=x(0)$, there is a unique $y \in \Lambda^{\infty}$ such that $\sigma^{d(\lambda)}(y)=x$; we often write $y=\lambda x$.

Definition 2.5 [Kumjian and Pask 2000, Definition 2.1]. Given a row-finite, sourcefree $k$-graph $\Lambda$, the associated path groupoid $\mathcal{G}_{\Lambda}$ is the groupoid associated to the equivalence relation on $\Lambda^{\infty}$ of "shift equivalence with lag". In other words,

$$
\mathcal{G}_{\Lambda}:=\left\{(x, n-m, y) \in \Lambda^{\infty} \times \mathbb{Z}^{k} \times \Lambda^{\infty}: n, m \in \mathbb{N}^{k}, \sigma^{n}(x)=\sigma^{m}(y)\right\}
$$

and $\mathcal{G}_{\Lambda}^{(0)}=\Lambda^{\infty}$, with $r(x, \ell, y)=x, s(x, \ell, y)=y$ and multiplication and inversion in $\mathcal{G}_{\Lambda}$ given by $(x, \ell, y)(y, m, z)=(x, \ell+m, z),(x, \ell, y)^{-1}=(y,-\ell, x)$.

When $\Lambda$ is a row-finite, source-free $k$-graph, Proposition 2.8 in [Kumjian and Pask 2000] tells us that the sets

$$
Z(\mu, \nu):=\{(\mu x, d(\mu)-d(\nu), v x): x(0)=s(\mu)=s(\nu)\}
$$

form a basis of compact open sets for a locally compact Hausdorff topology on $\mathcal{G}_{\Lambda}$ (in fact, with this topology $\mathcal{G}_{\Lambda}$ is an ample étale groupoid). 
To build a $C^{*}$-algebra out of a groupoid $\mathcal{G}$ we start by putting a $*$-algebra structure on $C_{c}(\mathcal{G})$, and to do this we need to integrate over the groupoid $\mathcal{G}$. A Haar system $\left\{\lambda^{u}\right\}_{u \in \mathcal{G}^{(0)}}$ (the groupoid analogue of Haar measure for groups; see [Renault 1980, Definition I.2.2]) will allow us to do this. Unlike in the group case, one cannot make existence or uniqueness statements about Haar systems for groupoids, so one usually starts by hypothesizing the existence of a fixed Haar system. For example, we obtain a Haar system $\left\{\lambda^{x}\right\}_{x \in \Lambda^{\infty}}$ on $\mathcal{G}_{\Lambda}$ by setting

$$
\lambda^{x}(E)=\#\left\{e \in E: e=(x, n, y) \text { for some } n \in \mathbb{Z}^{k}, y \in \Lambda^{\infty}\right\} .
$$

We will always use this Haar system on $\mathcal{G}_{\Lambda}$ in this paper.

Definition 2.6. Let $\mathcal{G}$ be a locally compact Hausdorff groupoid equipped with a Haar system $\left\{\lambda^{u}\right\}_{u \in \mathcal{G}^{(0)}}$ and a continuous 2-cocycle $\omega$. We define a *-algebra structure on $C_{c}(\mathcal{G})$ as follows: for $f, g \in C_{c}(\mathcal{G})$ let

$$
\begin{aligned}
f *_{\omega} g(a) & =\int_{\mathcal{G}_{s(a)}} f(a b) g\left(b^{-1}\right) \omega\left(a b, b^{-1}\right) d \lambda^{s(a)}(b), \\
f^{*}(a) & =\overline{f\left(a^{-1}\right) \omega\left(a, a^{-1}\right)} .
\end{aligned}
$$

From [Renault 1980, Proposition II.1.1] we know that the multiplication is welldefined (that is, that $f *_{\omega} g \in C_{c}(\mathcal{G})$ as claimed) and associative, and that $\left(f^{*}\right)^{*}=f$ so that the involution is involutive. The proof of associativity relies on the cocycle condition (2).

Given the fundamental role that the cocycle $\omega$ plays in the multiplication and involution on $C_{c}(\mathcal{G})$, we will often write $C_{c}(\mathcal{G}, \omega)$ to denote the set $C_{c}(\mathcal{G})$ equipped with the $*$-algebra structure of Definition 2.6. We define $C^{*}(\mathcal{G}, \omega)$ to be the completion of $C_{c}(\mathcal{G}, \omega)$ in the maximal $C^{*}$-norm, as described in Chapter II of [Renault 1980].

Definition 2.7. When $\mathcal{G}=\mathcal{G}_{\Lambda}$ is the groupoid associated to a row-finite $k$-graph $\Lambda$ with no sources, Lemma 6.3 of [Kumjian et al. 2015] explains how, given a cocycle $c \in \underline{Z}^{2}(\Lambda, \mathbb{T})$, we can construct a cocycle $\sigma_{c} \in Z^{2}\left(\mathcal{G}_{\Lambda}, \mathbb{T}\right)$. Then Corollary 7.8 of [Kumjian et al. 2015] shows that $C^{*}(\Lambda, c) \cong C^{*}\left(\mathcal{G}_{\Lambda}, \sigma_{c}\right)$. The construction of $\sigma_{c}$ is rather technical, but since we will need the details later, we present it here.

Lemma 6.6 of [Kumjian et al. 2015] establishes the existence of a subset

$$
\mathcal{P} \subseteq\{Z(\mu, \nu): s(\mu)=s(\nu)\}
$$

that partitions $\mathcal{G}_{\Lambda}$. In other words, every $a \in \mathcal{G}_{\Lambda}$ has exactly one representation of the form $a=\left(\mu_{a} x, d\left(\mu_{a}\right)-d\left(v_{a}\right), v_{a} x\right)$ with $Z\left(\mu_{a}, v_{a}\right) \in \mathcal{P}$. Note that if $(a, b) \in \mathcal{G}_{\Lambda}^{(2)}$, we need not have $\mu_{a}=\mu_{a b}$ or $v_{b}=v_{a b}$. However, given $(a, b) \in \mathcal{G}_{\Lambda}^{(2)}$, Lemma 6.3(i) of [Kumjian et al. 2015] shows that we can always find $y \in \Lambda^{\infty}$ and $\alpha, \beta, \gamma \in \Lambda$ 
such that

$$
\begin{aligned}
a & =\left(\mu_{a} \alpha y, d\left(\mu_{a}\right)-d\left(v_{a}\right), v_{a} \alpha y\right), \\
b & =\left(\mu_{b} \beta y, d\left(\mu_{b}\right)-d\left(v_{b}\right), v_{b} \beta y\right), \\
a b & =\left(\mu_{a b} \gamma y, d\left(\mu_{a b}\right)-d\left(v_{a b}\right), v_{a b} \gamma y\right) .
\end{aligned}
$$

Then, given a 2-cocycle $c$ on $\Lambda$, we define a 2-cocycle $\sigma_{c}$ on $\mathcal{G}_{\Lambda}$ by

$$
\sigma_{c}(a, b)=c\left(\mu_{a}, \alpha\right) c\left(\mu_{b}, \beta\right) c\left(v_{a b}, \gamma\right) \overline{c\left(v_{a}, \alpha\right) c\left(v_{b}, \beta\right) c\left(\mu_{a b}, \gamma\right)} .
$$

Since $c$ satisfies the cocycle condition (2), it's straightforward to check that $\sigma_{c}$ does also. Lemma 6.3 of [Kumjian et al. 2015] checks that $\sigma_{c}$ is well-defined and continuous, so we can construct the groupoid $C^{*}$-algebra $C^{*}\left(\mathcal{G}_{\Lambda}, \sigma_{c}\right)$ as outlined above. Corollary 7.8 of [Kumjian et al. 2015] tells us that $C^{*}\left(\mathcal{G}_{\Lambda}, \sigma_{c}\right) \cong C^{*}(\Lambda, c)$.

Theorem 6.5 of [Kumjian et al. 2015] establishes that different choices of partitions $\mathcal{P}$ give rise to cohomologous groupoid cocycles, and hence to isomorphic twisted groupoid $C^{*}$-algebras.

\section{Homotopies of cocycles}

In order to define a homotopy of groupoid 2-cocycles, we begin by observing that, given any locally compact Hausdorff groupoid $\mathcal{G}$, we can make $\mathcal{G} \times[0,1]$ into a locally compact Hausdorff groupoid by equipping it with the product topology and setting $(\mathcal{G} \times[0,1])^{(2)}:=\mathcal{G}^{(2)} \times[0,1]$. In other words, $(\mathcal{G} \times[0,1])^{(0)}=\mathcal{G}^{(0)} \times[0,1]$ and

$$
r(\gamma, t)=(r(\gamma), t), \quad s(\gamma, t)=(s(\gamma), t) .
$$

Moreover, if $\mathcal{G}$ has a Haar system $\left\{\lambda^{u}\right\}_{u \in \mathcal{G}^{(0)}}$, then setting $\lambda^{u, t}:=\lambda^{u}$ for every $t \in[0,1]$ gives rise to a Haar system on $\mathcal{G} \times[0,1]$. We will always use this Haar system on $\mathcal{G} \times[0,1]$ in this paper.

Definition 3.1 [Gillaspy 2015, Definition 2.12]. A homotopy of (2-)cocycles on a locally compact Hausdorff groupoid $\mathcal{G}$ is a 2-cocycle $\omega \in Z^{2}(\mathcal{G} \times[0,1], \mathbb{T})$. We say that two cocycles $\omega_{0}, \omega_{1} \in Z^{2}(\mathcal{G}, \mathbb{T})$ are homotopic if there exists a homotopy $\omega \in Z^{2}(\mathcal{G} \times[0,1], \mathbb{T})$ such that $\omega_{i}=\left.\omega\right|_{\mathcal{G} \times\{i\}}$ for $i=0,1$.

If $\omega$ is a homotopy of cocycles on $\mathcal{G}$ linking $\omega_{0}, \omega_{1}$, Theorem 3.3 below tells us that $C^{*}\left(\mathcal{G}, \omega_{0}\right)$ and $C^{*}\left(\mathcal{G}, \omega_{1}\right)$ are quotients of $C^{*}(\mathcal{G} \times[0,1], \omega)$. This is fundamental to the proof of our main result, Theorem 4.1.

Definition 3.2 [Williams 2007, Definition C.1]. Let $X$ be a locally compact Hausdorff space. A $C^{*}$-algebra $A$ is a $C_{0}(X)$-algebra if we have a $*$-homomorphism $\Phi: C_{0}(X) \rightarrow Z M(A)$ such that

$$
A=\overline{\operatorname{span}}\left\{\Phi(f) a: f \in C_{0}(X), a \in A\right\} .
$$

We usually write $f \cdot a$ for $\Phi(f) a$. 
If $A$ is a $C_{0}(X)$-algebra, then, for any $x \in X, \overline{\operatorname{span}} C_{0}(X \backslash x) \cdot A$ is an ideal $I_{x}$. We call $A_{x}:=A / I_{x}$ the fiber of $A$ at $x \in X$.

Theorem 3.3. Let $\omega$ be a homotopy of cocycles on a locally compact Hausdorff groupoid $\mathcal{G}$ with Haar system $\left\{\lambda^{u}\right\}_{u \in \mathcal{G}^{(0)}}$. Then $C^{*}(\mathcal{G} \times[0,1], \omega)$ is a $C([0,1])$ algebra, with fiber $C^{*}\left(\mathcal{G}, \omega_{t}\right)$ at $t \in[0,1]$.

Proof. We begin by checking that $C^{*}(\mathcal{G} \times[0,1], \omega)$ is a $C([0,1])$-algebra. For $f \in C([0,1]), \phi \in C_{c}(\mathcal{G} \times[0,1], \omega)$, define

$$
f \cdot \phi(a, t)=f(t) \phi(a, t) .
$$

It's not difficult to check that this action extends to a $*$-homomorphism

$$
\Phi: C([0,1]) \rightarrow Z M\left(C^{*}(\mathcal{G} \times[0,1], \omega)\right)
$$

such that $\|\Phi(f) \phi\| \leq\|f\|_{\infty}\|\phi\|$, or to check that

$$
\Phi(C([0,1])) \cdot C_{c}(\mathcal{G} \times[0,1], \omega)=C_{c}(\mathcal{G} \times[0,1], \omega)
$$

is dense in $C^{*}(\mathcal{G} \times[0,1], \omega)$. In other words, $\Phi$ makes $C^{*}(\mathcal{G} \times[0,1], \omega)$ into a $C([0,1])$-algebra as claimed.

Fix $t \in[0,1]$ and denote by $q_{t}: C_{c}(G \times[0,1], \omega) \rightarrow C_{c}\left(G, \omega_{t}\right)$ the evaluation map. Then $q_{t}$ is bounded by the $I$-norm (see [Renault 1980, Section II.1]), and hence extends to a surjective $*$-homomorphism $q_{t}: C^{*}(\mathcal{G} \times[0,1], \omega) \rightarrow C^{*}\left(\mathcal{G}, \omega_{t}\right)$. In other words, $C^{*}\left(\mathcal{G}, \omega_{t}\right)$ is a quotient of $C^{*}(\mathcal{G} \times[0,1], \omega)$. To see that $C^{*}\left(\mathcal{G}, \omega_{t}\right) \cong$ $C^{*}(\mathcal{G} \times[0,1], \omega)_{t}$, we need to check that ker $q_{t}=I_{t}$. A standard approximation argument will show that $\operatorname{ker} q_{t} \supseteq I_{t}$; thus, we will only detail the proof that ker $q_{t} \subseteq I_{t}$.

Note that the fiber algebra $C^{*}(\mathcal{G} \times[0,1], \omega)_{t} \cong C^{*}(\mathcal{G} \times[0,1], \omega) / I_{t}$ can be calculated as a completion $\overline{C_{c}(\mathcal{G} \times[0,1], \omega)}$ with respect to the norm given by

$$
\|f\|_{t}:=\sup \left\{\|L(f)\|: L\left(I_{t}\right)=0, L \text { is an } I \text {-norm-bounded representation }\right\} .
$$

Thus, to show that ker $q_{t} \subseteq I_{t}$, we will show that each such representation $L$ factors through $q_{t}$.

Given such a representation $L: C_{c}(\mathcal{G} \times[0,1], \omega) \rightarrow B(\mathcal{H})$, define $L^{\prime}: C_{c}\left(\mathcal{G}, \omega_{t}\right) \rightarrow$ $B(\mathcal{H})$ by $L^{\prime}\left(q_{t}(f)\right):=L(f)$. We claim that $L^{\prime}$ is an $I$-norm-bounded representation of $C_{c}\left(\mathcal{G}, \omega_{t}\right)$. To see this, it suffices to check that $L^{\prime}$ is well-defined and bounded.

Lemma 3.4. If $f, g \in C_{c}(\mathcal{G} \times[0,1], \omega)$ satisfy $q_{t}(f)=q_{t}(g)$, then the function $h=f-g \in C_{c}(\mathcal{G} \times[0,1], \omega)$ lies in $I_{t}$. Consequently, $L(f)=L(g)$ and $L^{\prime}$ is well-defined on $C_{c}\left(\mathcal{G}, \omega_{t}\right)$.

Proof. Let $\left\{f_{i}\right\}_{i \in I}$ be an approximate unit for $C_{0}([0,1] \backslash t)$ such that $f_{i}(s) \nearrow 1$ for every $s \neq t$; moreover, suppose that for each $i$ there exists a $\delta_{i}>0$ such that $f_{i}(s)=1$ if $|s-t| \geq \delta_{i}$. We will show that the $I$-norm $\left\|h-f_{i} h\right\|_{I}$ tends to 0 . Consequently, $h=\lim _{i} f_{i} h$ in $C^{*}(\mathcal{G} \times[0,1], \omega)$, so $h \in I_{t}$. 
For any $k \in C_{c}(\mathcal{G} \times[0,1], \omega)$, the axioms of a Haar system tell us that the function $(u, t) \mapsto \int|k(a, t)| d \lambda^{u, t}(a)$ is in $C_{0}\left(\mathcal{G}^{(0)} \times[0,1]\right)$. In particular, if we take $k$ to be a function that equals 1 where $h$ is nonzero and vanishes rapidly off $\operatorname{supp} h$, this shows us that $\phi(u, t):=\lambda^{u, t}(\operatorname{supp} h)$ is a pointwise limit of functions in $C_{0}\left(\mathcal{G}^{(0)} \times[0,1]\right)$, and hence is bounded. Let $K=\max \phi$.

Let $\epsilon>0$ be given. Since $h$ is compactly supported and since $h(a, t)=0$ for all $a \in \mathcal{G}$, we can choose a $\delta>0$ such that $|h(a, s)|<\epsilon / K$ for all $a \in \mathcal{G}$ whenever $|s-t|<\delta$, and we can choose a $j$ such that $i \geq j$ means $\delta_{i}<\delta$. Then, if $|s-t|<\delta$,

$$
\int_{\mathcal{G}^{u}}\left|h(a, s)-f_{i}(s) h(a, s)\right| d \lambda^{u}(a)=\left(1-f_{i}(s)\right) \int_{G^{u}}|h(a, s)| d \lambda^{u}(a)<1 \cdot \epsilon .
$$

On the other hand, if $|s-t| \geq \delta>\delta_{i}$, then $f_{i}(s)=1$ and

$$
\int_{\mathcal{G}^{u}}\left|h(a, s)-f_{i}(s) h(a, s)\right| d \lambda^{u}(a)=0
$$

for any $u \in \mathcal{G}^{(0)}$. In either case, given any $\epsilon>0$ we can always choose $j$ such that $i \geq j$ implies

$$
\begin{aligned}
\left\|h-f_{i} h\right\|_{I}=\max \left\{\sup _{s \in[0,1]} \sup _{u \in \mathcal{G}^{(0)}} \int_{\mathcal{G}^{u}}\left|h(a, s)-f_{i}(s) h(a, s)\right| d \lambda^{u}(a),\right. \\
\left.\sup _{s \in[0,1]} \sup _{u \in \mathcal{G}^{(0)}} \int_{\mathcal{G}^{u}}\left|h\left(a^{-1}, s\right)-f_{i}(s) h\left(a^{-1}, s\right)\right| d \lambda^{u}(a)\right\}<\epsilon .
\end{aligned}
$$

Since $\left\|h-f_{i} h\right\| \leq\left\|h-f_{i} h\right\|_{I}$ and $I_{t}$ is closed, it follows that $h \in I_{t}$ as desired, and so $L(h)=0$.

Having seen that $L^{\prime}$ is well-defined, we now proceed to show that it is bounded.

Lemma 3.5. For any fixed $f \in C_{c}(\mathcal{G} \times[0,1], \omega)$, the map $s \mapsto\left\|q_{s}(f)\right\|_{I}$ is continuous.

Proof. Fix $f \in C_{c}(\mathcal{G} \times[0,1], \omega)$ and fix $t \in[0,1]$. As in the proof of Lemma 3.4, let $K$ denote the supremum of the function $(u, s) \mapsto \lambda^{u, s}(\operatorname{supp} f)$. Since $f$ has compact support, given $\epsilon>0$ we can choose a $\delta$ such that

$$
|s-t|<\delta \Rightarrow|f(a, t)-f(a, s)|<\frac{\epsilon}{2 K} \quad \text { for all } a \in \mathcal{G} .
$$

Now, by definition of the $I$-norm, there exists a $u \in \mathcal{G}^{(0)}$ such that either

$$
\left\|q_{s}(f)\right\|_{I}<\int_{\mathcal{G}^{u}}|f(a, s)| d \lambda^{u}(a)+\frac{\epsilon}{2}
$$

or

$$
\left\|q_{s}(f)\right\|_{I}<\int_{\mathcal{G}^{u}}\left|f\left(a^{-1}, s\right)\right| d \lambda^{u}(a)+\frac{\epsilon}{2} .
$$


It follows that either

$$
\left\|q_{s}(f)\right\|_{I}<\int_{\mathcal{G}^{u}}|f(a, t)|+\frac{\epsilon}{2 K} d \lambda^{u}(a)+\frac{\epsilon}{2} \leq \int_{\mathcal{G}^{u}}|f(a, t)| d \lambda^{u}(a)+\epsilon
$$

or

$$
\left\|q_{s}(f)\right\|_{I}<\int_{\mathcal{G}^{u}}\left|f\left(a^{-1}, t\right)\right|+\frac{\epsilon}{2 K} d \lambda^{u}(a)+\frac{\epsilon}{2} \leq \int_{\mathcal{G}^{u}}\left|f\left(a^{-1}, t\right)\right| d \lambda^{u}(a)+\epsilon .
$$

Thus,

$$
\begin{aligned}
\left\|q_{s}(f)\right\|_{I} & <\max \left\{\int_{\mathcal{G}^{u}}|f(a, t)| d \lambda^{u}(a), \int_{\mathcal{G}^{u}}\left|f\left(a^{-1}, t\right)\right| d \lambda^{u}(a)\right\}+\epsilon \\
& \leq \max \left\{\sup _{u \in \mathcal{G}^{(0)}} \int_{\mathcal{G}^{u}}|f(a, t)| d \lambda^{u}(a), \sup _{u \in \mathcal{G}^{(0)}} \int_{\mathcal{G}^{u}}\left|f\left(a^{-1}, t\right)\right| d \lambda^{u}(a)\right\}+\epsilon \\
& =\left\|q_{t}(f)\right\|_{I}+\epsilon
\end{aligned}
$$

if $|s-t|<\delta$. Reversing the roles of $s$ and $t$ in the above argument tells us that

$$
|s-t|<\delta \Rightarrow\left|\left\|q_{s}(f)\right\|_{I}-\left\|q_{t}(f)\right\|_{I}\right|<\epsilon .
$$

Now we can finish the proof of Theorem 3.3. Set $S_{t}=\{\psi \in C([0,1]): \psi(t)=1\}$. For any $\psi \in S_{t}$ and any $f \in C_{c}(\mathcal{G} \times[0,1], \omega)$, we have

$$
\|L(\psi \cdot f)\|=\left\|L^{\prime}\left(q_{t}(\psi \cdot f)\right)\right\|=\left\|L^{\prime}\left(q_{t}(f)\right)\right\| .
$$

Consequently,

$$
\begin{aligned}
& \left\|L^{\prime}\left(q_{t}(f)\right)\right\|=\inf _{\psi \in S}\|L(\psi \cdot f)\| \leq \inf _{\psi}\|\psi \cdot f\|_{I} \\
& =\inf _{\psi} \max \left\{\sup _{s \in[0,1]} \sup _{u \in \mathcal{G}^{(0)}} \int|\psi(s) f(a, s)| d \lambda^{u}(a),\right. \\
& \left.\sup _{s \in[0,1]} \sup _{u \in \mathcal{G}^{(0)}} \int\left|\psi(s) f\left(a^{-1}, s\right)\right| d \lambda^{u}(a)\right\} \\
& =\inf _{\psi} \sup _{s \in[0,1]}\left\|q_{s}(\psi \cdot f)\right\|_{I} .
\end{aligned}
$$

Let $\epsilon>0$ be given. Choose a $\delta$ such that $|s-t|<\delta \Rightarrow\left|\left\|q_{s}(f)\right\|_{I}-\left\|q_{t}(f)\right\|_{I}\right|<\epsilon$; choose $\psi_{\epsilon} \in C([0,1])$ such that $\psi_{\epsilon}(t)=1$ and $|s-t| \geq \delta \Rightarrow \psi_{\epsilon}(s)=0$. Then, since $\psi_{\epsilon} \in S_{t}$, we have

$$
\left\|q_{s}\left(\psi_{\epsilon} \cdot f\right)\right\|_{I}=\psi_{\epsilon}(s)\left\|q_{s}(f)\right\|_{I}<\psi_{\epsilon}(s)\left(\left\|q_{t}(f)\right\|_{I}+\epsilon\right) \leq\left\|q_{t}(f)\right\|_{I}+\epsilon
$$

if $|s-t|<\delta$; otherwise we have $\left\|q_{s}\left(\psi_{\epsilon} \cdot f\right)\right\|_{I}=0$, and (3) still holds. 
Since we can find such a $\psi_{\epsilon}$ for any $\epsilon>0$, it follows that

$$
\begin{aligned}
\left\|L^{\prime}\left(q_{t}(f)\right)\right\| & \leq \inf _{\psi \in S_{t}} \sup _{s \in[0,1]}\left\|q_{s}(\psi \cdot f)\right\|_{I} \leq \inf _{\epsilon} \sup _{s}\left\|q_{s}\left(\psi_{\epsilon} \cdot f\right)\right\|_{I} \\
& \leq \inf _{\epsilon}\left\|q_{t}(f)\right\|_{I}+\epsilon=\left\|q_{t}(f)\right\|_{I} .
\end{aligned}
$$

The fact that $q_{t}$ is onto now tells us that $L^{\prime}$ is a bounded representation of $C_{c}\left(\mathcal{G}, \omega_{t}\right)$ as claimed. In other words, every representation $L$ of $C_{c}(\mathcal{G} \times[0,1], \omega)$ that kills $I_{t}$ also factors through $q_{t}$, so $\operatorname{ker} q_{t} \subseteq I_{t}$. This completes the proof that the fiber algebra $C^{*}(\mathcal{G} \times[0,1], \omega) / I_{t}$ of the $C([0,1])$-algebra $C^{*}(\mathcal{G} \times[0,1], \omega)$ is simply $C^{*}\left(\mathcal{G}, \omega_{t}\right)$.

In order to apply Theorem 3.3 to a homotopy of cocycles on a $k$-graph, we first need to define such a homotopy. Unlike for groupoids, there is no obvious way to make $\Lambda \times[0,1]$ into a higher-rank graph, so our definition of a homotopy of $k$-graph cocycles will look rather different than Definition 3.1 above. However, Proposition 3.8 below shows that the two definitions are compatible.

Definition 3.6. Let $\Lambda$ be a $k$-graph. A family $\left\{c_{t}\right\}_{t \in[0,1]}$ of 2-cocycles in $\underline{Z}^{2}(\Lambda, \mathbb{T})$ is a homotopy of (2-)cocycles on $\Lambda$ if for each pair $(\lambda, \mu) \in \Lambda^{* 2}$ the function $t \mapsto c_{t}(\lambda, \mu) \in \mathbb{T}$ is continuous.

Definition 3.7. Let $\left\{c_{t}\right\}_{t \in[0,1]}$ be a homotopy of cocycles on a $k$-graph $\Lambda$. Define $\omega \in Z^{2}\left(\mathcal{G}_{\Lambda} \times[0,1], \mathbb{T}\right)$ by

$$
\omega((a, t),(b, t))=\sigma_{c_{t}}(a, b),
$$

where $\sigma_{c_{t}}$ is the cocycle on $\mathcal{G}_{\Lambda}$ associated to $c_{t}$ as in Definition 2.7.

A moment's thought will reveal that $\omega$ satisfies the cocycle condition (2), since each $\sigma_{c_{t}}$ is a cocycle. Thus, in order to see that $\omega$ is a homotopy of cocycles on $\mathcal{G}_{\Lambda}$, we merely need to check that $\omega:\left(\mathcal{G}_{\Lambda} \times[0,1]\right)^{(2)} \rightarrow \mathbb{T}$ is continuous.

Proposition 3.8. The cocycle $\omega$ described in Definition 3.7 is continuous, and hence is a homotopy of groupoid cocycles on $\mathcal{G}_{\Lambda}$.

Proof. We will show that if $\left\{\left(a_{i}, b_{i}, t_{i}\right)\right\}_{i \in I} \subseteq \mathcal{G}_{\Lambda}^{(2)} \times[0,1]$ is a net which converges to $(a, b, t)$, then

$$
\omega\left(\left(a_{i}, t_{i}\right),\left(b_{i}, t_{i}\right)\right):=\sigma_{c_{t_{i}}}\left(a_{i}, b_{i}\right)=\sigma_{c_{t_{i}}}(a, b)
$$

for large enough $i$. Recall from Definition 2.7 that $\sigma_{c_{t_{i}}}(a, b)$ is a finite product of terms of the form $c_{t_{i}}(\mu, v)$ and their inverses, where the elements $\mu, v$ depend only on the elements $a, b$ and on the choice of partition $\mathcal{P}$ of $\mathcal{G}_{\Lambda}$ - but not on the 2-cocycle $c_{t_{i}}$. Thus, (4) and the continuity of the maps $t \mapsto c_{t}(\mu, v)$ will imply that $\omega\left(\left(a_{i}, t_{i}\right),\left(b_{i}, t_{i}\right)\right) \rightarrow \sigma_{c_{t}}(a, b)=\omega((a, t),(b, t))$. 
In what follows, we use the notation of Definition 2.7. If $\left(a_{i}, b_{i}, t_{i}\right) \rightarrow(a, b, t)$, then, for large enough $i$, we have $a_{i} \in Z\left(\mu_{a}, v_{a}\right), b_{i} \in Z\left(\mu_{b}, v_{b}\right)$, and in addition $a_{i} b_{i} \in Z\left(\mu_{a b}, v_{a b}\right)$. In other words, we can write

$$
\begin{gathered}
a=\left(\mu_{a} \alpha y, d\left(\mu_{a}\right)-d\left(v_{a}\right), v_{a} \alpha y\right), \quad a_{i}=\left(\mu_{a} \alpha_{i} y_{i}, d\left(\mu_{a}\right)-d\left(v_{a}\right), v_{a} \alpha_{i} y_{i}\right), \\
b=\left(\mu_{b} \beta y, d\left(\mu_{b}\right)-d\left(v_{b}\right), v_{b} \beta y\right), \quad b_{i}=\left(\mu_{b} \beta_{i} y_{i}, d\left(\mu_{b}\right)-d\left(v_{b}\right), v_{b} \beta_{i} y_{i}\right), \\
a b=\left(\mu_{a b} \gamma y, d\left(\mu_{a b}\right)-d\left(v_{a b}\right), v_{a b} \gamma y\right), \\
a_{i} b_{i}=\left(\mu_{a b} \gamma_{i} y_{i}, d\left(\mu_{a b}\right)-d\left(v_{a b}\right), v_{a b} \gamma_{i} y_{i}\right)
\end{gathered}
$$

for some $\alpha, \beta, \gamma, \alpha_{i}, \beta_{i}, \gamma_{i} \in \Lambda$ and $y, y_{i} \in \Lambda^{\infty}$.

Since $a_{i} \rightarrow a$ we must also have $\alpha_{i} y_{i} \rightarrow \alpha y$ in $\Lambda^{\infty}$. Thus, for large enough $i$, $\alpha_{i} y_{i} \in Z(\alpha):=\left\{\alpha y: y \in \Lambda^{\infty}, y(0)=s(\alpha)\right\}$ (see Proposition 2.8 of [Kumjian and Pask 2000]). It follows that

$$
\begin{gathered}
a_{i}=\left(\mu_{a} \alpha y_{i}^{\prime}, d\left(\mu_{a}\right)-d\left(v_{a}\right), v_{a} \alpha y_{i}^{\prime}\right), \quad b_{i}=\left(\mu_{b} \beta z_{i}^{\prime}, d\left(\mu_{b}\right)-d\left(v_{b}\right), v_{b} \beta z_{i}^{\prime}\right) \\
a_{i} b_{i}=\left(\mu_{a b} \gamma w_{i}^{\prime}, d\left(\mu_{a b}\right)-d\left(v_{a b}\right), v_{a b} \gamma w_{i}^{\prime}\right),
\end{gathered}
$$

where (since each pair $\left(a_{i}, b_{i}\right)$ is in $\mathcal{G}_{\Lambda}^{(2)}$ by hypothesis)

$$
v_{a} \alpha y_{i}^{\prime}=\mu_{b} \beta z_{i}^{\prime}, \quad \mu_{a} \alpha y_{i}^{\prime}=\mu_{a b} \gamma w_{i}^{\prime}, \quad v_{b} \beta z_{i}^{\prime}=v_{a b} \gamma w_{i}^{\prime} .
$$

Now, $v_{a} \alpha=\mu_{b} \beta$ by [Kumjian et al. 2015, Lemma 6.3], and thus $y_{i}^{\prime}=z_{i}^{\prime}$. A similar argument gives $z_{i}^{\prime}=w_{i}^{\prime}$ as well, so $y_{i}^{\prime}=z_{i}^{\prime}=w_{i}^{\prime}$. In other words, for large enough $i$,

$$
\begin{aligned}
\sigma_{c_{t_{i}}}\left(a_{i}, b_{i}\right) & =c_{t_{i}}\left(\mu_{a}, \alpha\right) c_{t_{i}}\left(\mu_{b}, \beta\right) c_{t_{i}}\left(v_{a b}, \gamma\right) \overline{c_{t_{i}}\left(v_{a}, \alpha\right) c_{t_{i}}\left(v_{b}, \beta\right) c_{t_{i}}\left(\mu_{a b}, \gamma\right)} \\
& =\sigma_{c_{t_{i}}}(a, b),
\end{aligned}
$$

as claimed. As observed in the first paragraph of the proof, it now follows that $\omega$ is a homotopy of cocycles on $\mathcal{G}_{\Lambda}$ as desired.

Corollary 3.9. Let $\left\{c_{t}\right\}$ be a homotopy of cocycles on a k-graph $\Lambda$, and define a cocycle $\omega$ on $\mathcal{G}_{\Lambda} \times[0,1]$ as in Definition 3.7. Then $C^{*}\left(\mathcal{G}_{\Lambda} \times[0,1], \omega\right)$ is a $C([0,1])$-algebra with fiber algebra $C^{*}\left(\mathcal{G}_{\Lambda}, \sigma_{c_{t}}\right) \cong C^{*}\left(\Lambda, c_{t}\right)$ at $t \in[0,1]$.

Proof. Proposition 3.8 tells us that $\omega$ is a homotopy of cocycles on $\mathcal{G}_{\Lambda}$. Theorem 3.3 tells us that the fiber over $t \in[0,1]$ of the $C([0,1])$-algebra $C^{*}\left(\mathcal{G}_{\Lambda} \times[0,1], \omega\right)$ is $C^{*}\left(\mathcal{G}_{\Lambda}, \sigma_{c_{t}}\right)$. The final isomorphism is provided by Corollary 7.8 of [Kumjian et al. 2015].

\section{The main theorem}

Theorem 4.1. Let $\Lambda$ be a row-finite $k$-graph with no sources and let $\left\{c_{t}\right\}_{t \in[0,1]}$ be a homotopy of cocycles on $\Lambda$. Then

$$
K_{*}\left(C^{*}\left(\Lambda, c_{0}\right)\right) \cong K_{*}\left(C^{*}\left(\Lambda, c_{1}\right)\right) .
$$


Moreover, this isomorphism preserves the $K$-theory class of the vertex projection $s_{v}$ for each $v \in \operatorname{Obj}(\Lambda)$.

We begin by proving a stronger version of Theorem 4.1 in the simpler case when the degree functor $d$ satisfies $d(\lambda)=\delta b(\lambda):=b(s(\lambda))-b(r(\lambda))$ for some function $b: \operatorname{Obj}(\Lambda) \rightarrow \mathbb{Z}^{k}$; this is Proposition 4.2 below. We then combine Proposition 4.2 with techniques from [Kumjian et al. 2013] to prove Theorem 4.1 in full generality.

The AF case. If $(\Lambda, d)$ is a $k$-graph such that $d=\delta b$, then Lemma 8.4 of [Kumjian et al. 2015] tells us that $C^{*}(\Lambda, c)$ and $C^{*}(\Lambda)$ are both AF-algebras, with the same approximating subalgebras and multiplicities of partial inclusions. Consequently, $C^{*}(\Lambda, c) \cong C^{*}(\Lambda)$. In order to fix notation for what follows, we describe this isomorphism in some detail.

Lemma 3.1 of [Kumjian and Pask 2000] shows that if $\Lambda$ is a row-finite, sourcefree $k$-graph, then $\left\{s_{\lambda} s_{\mu}^{*}: s(\lambda)=s(\mu)\right\}$ spans a dense $*$-subalgebra of $C^{*}(\Lambda)$. Moreover, when $d=\delta b$, Lemma 5.4 of [Kumjian and Pask 2000] tells us that $\left\{s_{\lambda} s_{\mu}^{*}: b(s(\lambda))=b(s(\mu))=n\right\}$ forms a collection of matrix units for the subalgebra

$$
A_{n}=\overline{\operatorname{span}}\left\{s_{\lambda} s_{\mu}^{*}: b(s(\lambda))=b(s(\mu))=n\right\} \cong \bigoplus_{b(v)=n} \mathcal{K}\left(\ell^{2}\left(s^{-1}(v)\right)\right) .
$$

Observe that we can think of $A_{n}$ as a subalgebra of $C^{*}(\Lambda)$ or of $C^{*}(\Lambda, c)$. In fact, these subalgebras allow us to exhibit $C^{*}(\Lambda, c)$ and $C^{*}(\Lambda)$ as AF-algebras:

$$
C^{*}(\Lambda, c)=\underline{\lim }\left(A_{n}, \phi_{m, n}^{c}\right) \quad \text { and } \quad C^{*}(\Lambda)=\underline{\lim }\left(A_{n}, \phi_{m, n}\right),
$$

where the connecting maps $\phi_{m, n}, \phi_{m, n}^{c}: A_{n} \rightarrow A_{m}$ are given by

$$
\begin{aligned}
\phi_{m, n}^{c}\left(s_{\lambda} s_{\mu}^{*}\right) & =\sum_{\substack{r(\alpha)=s(\lambda) \\
b(s(\alpha))=m}} c(\lambda, \alpha) \overline{c(\mu, \alpha)} s_{\lambda \alpha} s_{\mu \alpha}^{*}, \\
\phi_{m, n}\left(s_{\lambda} s_{\mu}^{*}\right) & =\sum_{\substack{r(\alpha)=s(\lambda) \\
b(s(\alpha))=m}} s_{\lambda \alpha} s_{\mu \alpha}^{*} .
\end{aligned}
$$

We can now describe explicitly the isomorphism $C^{*}(\Lambda, c) \cong C^{*}(\Lambda)$. As in Theorem 4.2 of [Kumjian et al. 2013], write $\mathbb{1}$ for $(1, \ldots, 1) \in \mathbb{N}^{k}$, and define $\kappa: \Lambda \rightarrow \mathbb{T}$ by

$$
\kappa(\lambda)= \begin{cases}1 & d(\lambda) \nsupseteq \mathbb{1}, \\ \kappa(\mu) c(\mu, \alpha) & d(\alpha)=\mathbb{1} \text { and } \lambda=\mu \alpha .\end{cases}
$$

For $n \in \mathbb{Z}^{k}$, let $U_{n}=\sum_{b(s(\lambda))=n} \kappa(\lambda) s_{\lambda} s_{\lambda}^{*} \in U\left(M\left(A_{n}\right)\right)$. A quick computation will show that, for any $\lambda, \mu$ with $s_{\lambda} s_{\mu}^{*} \in A_{n}$,

$$
\operatorname{Ad} U_{n}\left(s_{\lambda} s_{\mu}^{*}\right)=\kappa(\lambda) \overline{\kappa(\mu)} s_{\lambda} s_{\mu}^{*} .
$$


Moreover, the factorization property tells us that, for any $h \in \mathbb{Z}$,

$$
\phi_{(h+1) \mathbb{1}, h \mathbb{1}}^{c} \circ \operatorname{Ad} U_{h \mathbb{1}}=\operatorname{Ad} U_{(h+1) \mathbb{1}} \circ \phi_{(h+1) \mathbb{1}, h \mathbb{1}} .
$$

In other words, $\operatorname{Ad} U_{*}$ intertwines the connecting maps $\phi_{m, n}^{c}, \phi_{m, n}$, and hence implements the isomorphism $C^{*}(\Lambda) \rightarrow C^{*}(\Lambda, c)$.

We can now use this isomorphism to prove that a homotopy of cocycles on $\Lambda$ gives rise to a trivial continuous field when $d=\delta b$.

Proposition 4.2. Let $(\Lambda, d)$ be a row-finite, source-free $k$-graph such that $d=\delta b$ for some function $b: \operatorname{Obj}(\Lambda) \rightarrow \mathbb{Z}^{k}$, let $\left\{c_{t}\right\}_{t \in[0,1]}$ be a homotopy of cocycles on $\Lambda$, and let $\omega$ be the cocycle on $\mathcal{G}_{\Lambda} \times[0,1]$ associated to $\left\{c_{t}\right\}_{t \in[0,1]}$ as in Definition 3.7. We have an isomorphism of $C([0,1])$-algebras

$$
C^{*}\left(\mathcal{G}_{\Lambda} \times[0,1], \omega\right) \cong C^{*}\left(\mathcal{G}_{\Lambda} \times[0,1]\right) \cong C([0,1]) \otimes C^{*}(\Lambda) .
$$

Proof. Recall that

$$
C^{*}\left(\mathcal{G}_{\Lambda} \times[0,1]\right)_{t} \cong C^{*}\left(\mathcal{G}_{\Lambda}, \sigma_{c_{t}}\right) \cong C^{*}\left(\Lambda, c_{t}\right) \cong C^{*}(\Lambda)
$$

if $d=\delta b$. Thus, the $C([0,1])$-algebras $C^{*}\left(\mathcal{G}_{\Lambda} \times[0,1], \omega\right)$ and $C([0,1]) \otimes C^{*}(\Lambda)$ have isomorphic fibers over each point $t \in[0,1]$.

In order to prove the proposition, we need to show that these isomorphisms $C^{*}\left(\mathcal{G}_{\Lambda}, \sigma_{c_{t}}\right) \cong C^{*}(\Lambda)$ vary continuously in $t$, so that they patch together to give us an isomorphism of $C([0,1])$-algebras $C^{*}\left(\mathcal{G}_{\Lambda} \times[0,1], \omega\right) \cong C([0,1]) \otimes C^{*}(\Lambda)$.

For each $t \in[0,1]$, let $\pi^{t}: C^{*}\left(\Lambda, c_{t}\right) \rightarrow C^{*}\left(\mathcal{G}_{\Lambda}, \sigma_{c_{t}}\right)$ denote the isomorphism described in Theorem 6.7 of [Kumjian et al. 2015]. Let $\pi: C^{*}(\Lambda) \rightarrow C^{*}\left(\mathcal{G}_{\Lambda}\right)$ denote the equivalent isomorphism for the case of a trivial cocycle $c$. For each $n \in \mathbb{Z}^{k}$, write $U_{n}^{t}$ for the unitary $U_{n}^{t}: A_{n} \rightarrow A_{n}$ associated to the cocycle $c_{t}$ as above. Setting

$$
\Psi_{t}:=\pi^{t} \circ \operatorname{Ad} U_{*}^{t} \circ \pi^{-1}
$$

consequently gives an isomorphism of $C^{*}$-algebras $\Psi_{t}: C^{*}\left(\mathcal{G}_{\Lambda}\right) \rightarrow C^{*}\left(\mathcal{G}_{\Lambda}, \sigma_{c_{t}}\right)$.

We claim that $\Psi:=\left\{\Psi_{t}\right\}_{t \in[0,1]}$ defines an isomorphism of $C([0,1])$-algebras

$$
\Psi: C^{*}\left(\mathcal{G}_{\Lambda} \times[0,1]\right) \rightarrow C^{*}\left(\mathcal{G}_{\Lambda} \times[0,1], \omega\right) .
$$

In order to prove this assertion, we begin by writing down an explicit formula for $\Psi_{t}$ on the characteristic functions $1_{Z(\mu, v)} \in C_{c}\left(\mathcal{G}_{\Lambda}\right)$ where $Z(\mu, v) \in \mathcal{P}$ and where $\mathcal{P}$ is the partition of $\mathcal{G}_{\Lambda}$ described in Lemma 6.6 of [Kumjian et al. 2015].

Recall that the value of $\sigma_{c_{t}}(a, b)$ depends only on the sets $Z(\mu, v) \in \mathcal{P}$ containing the points $a, b$, and $a b$ in $\mathcal{G}_{\Lambda}$. Moreover, the proof of [Kumjian et al. 2015, Theorem 6.7] establishes that, if $1_{Z(\mu, \nu)}$ denotes the characteristic function 
on $Z(\mu, v) \subseteq \mathcal{G}_{\Lambda}$, and if we write $a \in Z(\mu, \nu)$ as $a=b d$ where $b \in Z(\mu, s(\mu))$, $d \in Z(s(v), v)$, then

$$
\pi^{t}\left(s_{\mu} s_{v}^{*}\right)(a)=1_{Z(\mu, v)}(a) \sigma_{c_{t}}(b, d) \overline{\sigma_{c_{t}}\left(d^{-1}, d\right)}=1_{Z(\mu, v)}(a) \overline{\sigma_{c_{t}}\left(b d, d^{-1}\right)} .
$$

In addition, we have $Z(\mu, s(\mu)) \in \mathcal{P}$ for all $\mu \in \Lambda$ by Lemma 6.6 of [Kumjian et al. 2015]. If we also have $Z(\mu, v) \in \mathcal{P}$, then the elements $\alpha, \beta, \gamma$ in the formula for $\sigma_{c_{t}}\left(b d, d^{-1}\right)$ given in Definition 2.7 are all units, so, for any $t$, we have $\sigma_{c_{t}}\left(b d, d^{-1}\right)=1$ by our hypothesis that any cocycle $c$ satisfies the equality $c(\lambda, s(\lambda))=c(r(\lambda), \lambda)=1$. Thus,

$$
Z(\mu, v) \in \mathcal{P} \Rightarrow \pi^{t}\left(s_{\mu} s_{v}^{*}\right)=1_{Z(\mu, v)} \Rightarrow \Psi_{t}\left(1_{Z(\mu, v)}\right)=\kappa_{t}(\mu) \overline{\kappa_{t}(v)} 1_{Z(\mu, v)} .
$$

Now observe that each $f \in C_{c}\left(\mathcal{G}_{\Lambda} \times[0,1]\right)$ can be written as a finite sum $f(a, t)=\sum_{i \in N} f_{i}(a, t)$, where, for all $i$, we have $f_{i} \in C\left(Z\left(\mu_{i}, v_{i}\right) \times[0,1]\right)$ and $Z\left(\mu_{i}, v_{i}\right) \in \mathcal{P}$. Consequently, on $C_{c}\left(\mathcal{G}_{\Lambda} \times[0,1]\right)$, our map $\Psi$ becomes

$$
\Psi\left(\sum_{i \in N} f_{i}\right)(a, t)=\sum_{i \in N} \Psi_{t}\left(f_{i}(\cdot, t)\right)(a)=\sum_{i \in N} \kappa_{t}\left(\mu_{i}\right) \overline{\kappa_{t}\left(v_{i}\right)} f_{i}(a, t) ;
$$

the fact that all the sums are finite implies that $\Psi$ takes $C_{c}\left(\mathcal{G}_{\Lambda} \times[0,1]\right)$ onto $C_{c}\left(\mathcal{G}_{\Lambda} \times[0,1]\right)$.

Since $\Psi$ is evidently $C([0,1])$-linear and is a $*$-isomorphism in each fiber, Proposition C.10 of [Williams 2007] tells us that $\Psi$ is norm-preserving. Moreover, $\Psi$ is a $*$-homomorphism since the operations in $C_{c}\left(\mathcal{G}_{\Lambda} \times[0,1]\right)$ preserve the fiber over $t \in[0,1]$, and each $\Psi_{t}$ is a $*$-homomorphism.

In other words, $\Psi$ extends to an isomorphism of $C([0,1])$-algebras

$$
\Psi: C^{*}\left(\mathcal{G}_{\Lambda} \times[0,1]\right) \cong C^{*}\left(\mathcal{G}_{\Lambda} \times[0,1], \omega\right) .
$$

A straightforward check will establish that the identity map on $C_{c}\left(\mathcal{G}_{\Lambda} \times[0,1]\right)$ induces an isomorphism id : $C^{*}\left(\mathcal{G}_{\Lambda} \times[0,1]\right) \rightarrow C\left([0,1], C^{*}\left(\mathcal{G}_{\Lambda}\right)\right)$ of $C([0,1])$ algebras; the isomorphism $C^{*}\left(\mathcal{G}_{\Lambda}\right) \cong C^{*}(\Lambda)$ of [Kumjian and Pask 2000, Corollary 3.5(i)] now finishes the proof.

Remark 4.3. Note that $\Psi$ induces an isomorphism $\Phi: C([0,1]) \otimes C^{*}(\Lambda) \rightarrow$ $C^{*}\left(\mathcal{G}_{\Lambda} \times[0,1], \omega\right)$ as follows. If $Z(\mu, v) \in \mathcal{P}$ and $f \in C([0,1])$, then

$$
\Phi\left(f \otimes s_{\mu} s_{v}^{*}\right)(x, t)=f(t) 1_{Z(\mu, v)}(x) \kappa_{t}(\mu) \overline{\kappa_{t}(v)} .
$$

Remark 4.4. Since evaluation at $t \in[0,1]$ induces a homotopy equivalence between $C\left([0,1], C^{*}(\Lambda)\right)$ and $C^{*}(\Lambda)$, the isomorphism established in the previous proposition implies that evaluation at $t$ also induces a homotopy equivalence between $C^{*}\left(\mathcal{G}_{\Lambda} \times[0,1], \omega\right)$ and its fiber algebra $C^{*}\left(\mathcal{G}_{\Lambda}, \sigma_{c_{t}}\right)$ when $d=\delta b$. 
To leverage Proposition 4.2 into the proof of Theorem 4.1, we will use the skew-product $k$-graphs $\Lambda \times_{d} \mathbb{Z}^{k}$.

Definition 4.5 [Kumjian and Pask 2000, Definition 5.1]. Given a $k$-graph $(\Lambda, d)$, the skew-product $k$-graph $\Lambda \times_{d} \mathbb{Z}^{k}$ is the set $\Lambda \times \mathbb{Z}^{k}$, with the structure maps

$$
r(\lambda, n)=(r(\lambda), n), \quad s(\lambda, n)=(s(\lambda), n+d(\lambda)), \quad d(\lambda, n)=d(\lambda),
$$

and with multiplication given by $(\lambda, n)(\mu, n+d(\lambda))=(\lambda \mu, n)$ for $(\lambda, \mu) \in \Lambda^{* 2}$.

Observe that the function $b:\left(\Lambda \times_{d} \mathbb{Z}^{k}\right)^{(0)}=\Lambda^{(0)} \times \mathbb{Z}^{k} \rightarrow \mathbb{Z}^{k}$ given by $b(v, n)=n$ satisfies $\delta b=d$ on $\Lambda \times_{d} \mathbb{Z}^{k}$. Moreover, if $\Lambda$ is row-finite and source-free, then so is $\Lambda \times_{d} \mathbb{Z}^{k}$.

We can now complete the proof of Theorem 4.1.

Proof of Theorem 4.1. Let $\phi: \Lambda \times_{d} \mathbb{Z}^{k} \rightarrow \Lambda$ be the projection onto the first coordinate: $\phi(\lambda, n)=\lambda$. A cocycle $c$ on $\Lambda$ induces a cocycle $c \circ \phi$ on the skew-product $k$-graph $\Lambda \times_{d} \mathbb{Z}^{k}$ :

$$
c \circ \phi((\lambda, n),(\mu, n+d(\lambda))):=c(\lambda, \mu)
$$

whenever $(\lambda, \mu) \in \Lambda^{* 2}$. Note that if $\left\{c_{t}\right\}_{t \in[0,1]}$ is a homotopy of cocycles on $\Lambda$ then $\left\{c_{t} \circ \phi\right\}_{t}$ is also a homotopy of cocycles on $\Lambda \times_{d} \mathbb{Z}^{k}$.

If $\omega$ is the homotopy of cocycles on $\mathcal{G}_{\Lambda \times{ }_{d} \mathbb{Z}^{k}}$ associated to the homotopy $\left\{c_{t}\right\}_{t \in[0,1]}$ of cocycles on $\Lambda$, then Proposition 4.2 tells us that

$$
C^{*}\left(\mathcal{G}_{\Lambda \times_{d} \mathbb{Z}^{k}} \times[0,1], \omega\right) \cong C([0,1]) \otimes C^{*}\left(\Lambda \times_{d} \mathbb{Z}^{k}\right) .
$$

Now we define an action of $\mathbb{Z}^{k}$ on $C([0,1]) \otimes C^{*}\left(\Lambda \times_{d} \mathbb{Z}^{k}\right)$ by setting

$$
f \otimes s_{\lambda, n} \cdot m:=f \otimes s_{\lambda, n+m} .
$$

To see that this formula gives a well-defined action of $\mathbb{Z}^{k}$ on $C([0,1]) \otimes C^{*}\left(\Lambda \times_{d} \mathbb{Z}^{k}\right)$, one checks first that, for each $m \in \mathbb{Z}^{k}$, the set $\left\{s_{\lambda, m+n}: \lambda \in \Lambda, n \in \mathbb{Z}^{k}\right\}$ is a collection of partial isometries satisfying the defining axioms (CK1)-(CK4) for $C^{*}\left(\Lambda \times_{d} \mathbb{Z}^{k}\right)$. Consequently, the universal property of $C^{*}\left(\Lambda \times_{d} \mathbb{Z}^{k}\right)$ implies that, for each fixed $m \in \mathbb{Z}^{k}$, the map $s_{\lambda, n} \mapsto s_{\lambda, n+m}$ determines a $*$-homomorphism

$$
\alpha_{m}: C^{*}\left(\Lambda \times_{d} \mathbb{Z}^{k}\right) \rightarrow C^{*}\left(\Lambda \times_{d} \mathbb{Z}^{k}\right) .
$$

Each $\alpha_{m}$ is invertible with inverse $\alpha_{-m}$; it follows that $m \mapsto \alpha_{m}$ defines a group action of $\mathbb{Z}^{k}$ on $C^{*}\left(\Lambda \times_{d} \mathbb{Z}^{k}\right)$. Thus, (8) describes a well-defined action id $\otimes \alpha$ of $\mathbb{Z}^{k}$ on $C([0,1]) \otimes C^{*}\left(\Lambda \times_{d} \mathbb{Z}^{k}\right)$, given by $m \mapsto \mathrm{id} \otimes \alpha_{m}$. The fact that the degree map on $\Lambda \times_{d} \mathbb{Z}^{k}$ is a coboundary now allows us to combine the action id $\otimes \alpha$ with the isomorphism $\Phi: C([0,1]) \otimes C^{*}\left(\Lambda \times_{d} \mathbb{Z}^{k}\right) \rightarrow C^{*}\left(\mathcal{G}_{\Lambda \times_{d} \mathbb{Z}^{k}} \times[0,1], \omega\right)$ of Remark 4.3 to obtain an action $\beta$ of $\mathbb{Z}^{k}$ on $C^{*}\left(\mathcal{G}_{\Lambda \times_{d} \mathbb{Z}^{k}} \times[0,1], \omega\right)$ :

$$
\beta_{n}\left(\Phi\left(f \otimes s_{\mu, m} s_{v, m+d(\mu)-d(v)}^{*}\right)\right):=\Phi\left(\mathrm{id} \otimes \alpha_{n}\left(f \otimes s_{\mu, m} s_{v, m+d(\mu)-d(v)}^{*}\right)\right) .
$$


Moreover, since both id $\otimes \alpha$ and $\Phi$ (and hence $\beta$ ) fix $C([0,1])$ by construction, Lemma 5.3 of [Kumjian et al. 2013] tells us that the crossed product

$$
C^{*}\left(\mathcal{G}_{\Lambda \times_{d} \mathbb{Z}^{k}} \times[0,1], \omega\right) \rtimes_{\beta} \mathbb{Z}^{k} \cong\left(C([0,1]) \otimes C^{*}\left(\Lambda \times_{d} \mathbb{Z}^{k}\right)\right) \rtimes_{\mathrm{id} \otimes \alpha} \mathbb{Z}^{k}
$$

is a $C([0,1])$-algebra with fiber $C^{*}\left(\mathcal{G}_{\Lambda \times \mathbb{Z}^{k}}, \sigma_{c_{t} \circ \phi}\right) \rtimes_{\beta_{t}} \mathbb{Z}^{k}$, where

$$
\begin{aligned}
\left(\beta_{t}\right)_{n}\left(\Phi_{t}\left(s_{\mu, m} s_{v, m+d(\mu)-d(v)}^{*}\right)\right) & =\Phi_{t}\left(\alpha_{n}\left(s_{(\mu, m)} s_{(v, m+d(\mu)-d(v))}^{*}\right)\right) \\
& =\kappa_{t}(\mu) \overline{\kappa_{t}(v)} 1_{Z((\mu, m+n),(v, m+n+d(\mu)-d(v)))}
\end{aligned}
$$

whenever $Z((\mu, m+n),(\nu, m+n+d(\mu)-d(\nu)))$ is in the partition $\mathcal{P}$ of $\mathcal{G}_{\Lambda \times_{d} \mathbb{Z}^{k}}$ that we used in the proof of Proposition 4.2.

Recall that we have a homotopy equivalence $q_{t}: C^{*}\left(\mathcal{G}_{\Lambda \times_{d} \mathbb{Z}^{k}} \times[0,1], \omega\right) \rightarrow$ $C^{*}\left(\mathcal{G}_{\Lambda \times \mathbb{Z}^{k}}, \sigma_{c_{t}}\right)$. A computation will show that $q_{t}$ is equivariant with respect to the actions $\beta, \beta_{t}$ of $\mathbb{Z}^{k}$; thus, Theorem 5.1 of [Kumjian et al. 2013] tells us that

$$
K_{*}\left(C^{*}\left(\mathcal{G}_{\Lambda \times_{d} \mathbb{Z}^{k}} \times[0,1], \omega\right) \rtimes_{\beta} \mathbb{Z}^{k}\right) \cong K_{*}\left(C^{*}\left(\mathcal{G}_{\Lambda \times_{d} \mathbb{Z}^{k}}, \sigma_{c_{t} \circ \phi}\right) \rtimes_{\beta_{t}} \mathbb{Z}^{k}\right) .
$$

Thanks to Lemma 5.2 of [Kumjian et al. 2013], we know that

$$
C^{*}\left(\Lambda \times_{d} \mathbb{Z}^{k}, c_{t} \circ \phi\right) \rtimes_{l t} \mathbb{Z}^{k} \sim_{M E} C^{*}\left(\Lambda, c_{t}\right),
$$

where $l t_{m}\left(s_{\lambda, n}\right)=s_{\lambda, n+m}$. To make use of this result, we need to show that $\beta_{t}$ induces the action $l t$ on $C^{*}\left(\Lambda \times_{d} \mathbb{Z}^{k}, c_{t} \circ \phi\right)$.

Recall from the proof of Proposition 4.2 that $\pi^{t}\left(s_{\lambda, m}\right)=1_{Z((\lambda, m),(s(\lambda), m+s(\lambda)))}$, since $Z((\lambda, m),(s(\lambda), m+s(\lambda))) \in \mathcal{P}$ always. Observe that

$$
C^{*}\left(\mathcal{G}_{\Lambda \times_{d} \mathbb{Z}^{k}}, \sigma_{c_{t} \circ \phi}\right) \rtimes_{\beta_{t}} \mathbb{Z}^{k} \cong C^{*}\left(\Lambda \times_{d} \mathbb{Z}^{k}, c_{t} \circ \phi\right) \rtimes_{\gamma_{t}} \mathbb{Z}^{k},
$$

where

$$
\begin{aligned}
\left(\gamma_{t}\right)_{n}\left(s_{\lambda, m}\right): & =\left(\pi^{t}\right)^{-1}\left(\left(\beta_{t}\right)_{n}\left(\pi^{t}\left(s_{\lambda, m}\right)\right)\right)=\left(\pi^{t}\right)^{-1}\left(\beta_{t}\right)_{n}\left(1_{Z((\lambda, m),(s(\lambda), m))}\right) \\
& =\left(\pi^{t}\right)^{-1}\left(\beta_{t}\right)_{n}\left(\Phi_{t}\left(\overline{\kappa_{t}(\lambda)} s_{(\lambda, m)}\right)\right)=\left(\pi^{t}\right)^{-1}\left(\Phi_{t}\left(\alpha_{n}\left(\overline{\kappa_{t}(\lambda)} s_{\lambda, m}\right)\right)\right) \\
& =\left(\pi^{t}\right)^{-1}\left(\Phi_{t}\left(\overline{\kappa_{t}(\lambda)} s_{\lambda, m+n}\right)\right)=\left(\pi^{t}\right)^{-1}\left(1_{Z((\lambda, m+n),(s(\lambda), m+n))}\right) \\
& =s_{\lambda, m+n} .
\end{aligned}
$$

It follows that the action $\left(\gamma_{t}\right)$ induced by $\beta_{t}$ agrees with $l t$, as desired. Now, the Morita equivalence of Lemma 5.2 of [Kumjian et al. 2013] and (10) tell us that

$$
C^{*}\left(\mathcal{G}_{\Lambda \times_{d} \mathbb{Z}^{k}}, \sigma_{c_{t} \circ \phi}\right) \rtimes_{\beta_{t}} \mathbb{Z}^{k} \sim_{M E} C^{*}\left(\Lambda, c_{t}\right) .
$$

Combining (9) and (11) now yields

$$
K_{*}\left(C^{*}\left(\Lambda, c_{t}\right)\right) \cong K_{*}\left(C^{*}\left(\mathcal{G}_{\Lambda \times_{d} \mathbb{Z}^{k}} \times[0,1], \omega\right) \rtimes_{\beta} \mathbb{Z}^{k}\right)
$$


for any $t \in[0,1]$. It follows that, if $\left\{c_{t}\right\}_{t \in[0,1]}$ is a homotopy of cocycles on a row-finite $k$-graph $\Lambda$ with no sources, then, for any $s, t \in[0,1]$,

$$
K_{*}\left(C^{*}\left(\Lambda, c_{t}\right)\right) \cong K_{*}\left(C^{*}\left(\Lambda, c_{s}\right)\right) .
$$

It remains to show that this isomorphism preserves the $K$-theory class of each vertex projection $s_{v}$. Essentially, this follows because the cocycles $c_{t}$, and thus the functions $\kappa_{t}$, are all trivial on any $v \in \operatorname{Obj}(\Lambda)$.

To be precise, let $v \in \operatorname{Obj}(\Lambda)$ and define $f_{v} \in C_{c}\left(\mathbb{Z}^{k}, C_{c}\left(\mathcal{G}_{\Lambda \times_{d} \mathbb{Z}^{k}} \times[0,1]\right)\right) \subseteq$ $C^{*}\left(\mathcal{G}_{\Lambda \times_{d} \mathbb{Z}^{k}} \times[0,1], \omega\right) \rtimes_{\beta} \mathbb{Z}^{k}$ by

$$
f_{v}(n)(a, t)= \begin{cases}1 & a \in Z_{(v, 0),(v, 0)} \text { and } n=0, \\ 0 & \text { else. }\end{cases}
$$

Then the projection $q_{t} \rtimes \operatorname{id}\left(f_{v}\right)$ of $f_{v}$ onto the fiber algebra $C^{*}\left(\mathcal{G}_{\Lambda \times_{d} \mathbb{Z}^{k}}, \omega_{t}\right) \rtimes_{\beta_{t}} \mathbb{Z}^{k}$ is independent of the choice of $t \in[0,1]$ :

$$
q_{t} \rtimes \operatorname{id}\left(f_{v}\right)(n)(a)= \begin{cases}1 & a \in Z_{(v, 0),(v, 0)} \text { and } n=0, \\ 0 & \text { else }\end{cases}
$$

for any $t \in[0,1]$. Moreover, the isomorphism $\Phi_{t}: C^{*}\left(\Lambda \times_{d} \mathbb{Z}^{k}, c_{t} \circ \phi\right) \rightarrow$ $C^{*}\left(\mathcal{G}_{\Lambda \times_{d} \mathbb{Z}^{k}}, \sigma_{c_{t} \circ \phi}\right)$ of Remark 4.3 satisfies

$$
\Phi_{t} \rtimes \operatorname{id}\left(j\left(s_{(v, 0)}\right)\right)=q_{t} \rtimes \operatorname{id}\left(f_{v}\right),
$$

where $j: C^{*}\left(\Lambda \times_{d} \mathbb{Z}^{k}, c_{t} \circ \phi\right) \rightarrow C^{*}\left(\Lambda \times_{d} \mathbb{Z}^{k}, c_{t} \circ \phi\right) \rtimes_{l t} \mathbb{Z}^{k}$ is the canonical embedding of $C^{*}\left(\Lambda \times_{d} \mathbb{Z}^{k}, c_{t} \circ \phi\right)$ into the crossed product.

The fact that the Morita equivalence $C^{*}\left(\Lambda, c_{t}\right) \sim_{M E} C^{*}\left(\Lambda \times_{d} \mathbb{Z}^{k}, c_{t} \circ \phi\right) \rtimes_{l t} \mathbb{Z}^{k}$ takes $s_{v} \in C^{*}\left(\Lambda, c_{t}\right)$ to $j\left(s_{(v, 0)}\right)$ (see Lemma 5.2 in [Kumjian et al. 2013]) thus implies that our $K$-theoretic isomorphism $K_{*}\left(C^{*}\left(\mathcal{G}_{\Lambda \times_{d} \mathbb{Z}^{k}} \times[0,1], \omega\right) \rtimes_{\beta} \mathbb{Z}^{k}\right) \rightarrow$ $K_{*}\left(C^{*}\left(\Lambda, c_{t}\right)\right)$, which is given by the composition of the Morita equivalence (11) with the $*$-homomorphism

$$
\begin{aligned}
q_{t} \rtimes \mathrm{id}: C^{*}\left(\mathcal{G}_{\Lambda \times_{d} \mathbb{Z}^{k}} \times[0,1], \omega\right) \rtimes_{\beta} \mathbb{Z}^{k} \rightarrow C^{*}\left(\mathcal{G}_{\Lambda \times_{d} \mathbb{Z}^{k}}, \omega_{t}\right) \rtimes_{\beta_{t}} \mathbb{Z}^{k} \\
\\
\cong C^{*}\left(\Lambda \times_{d} \mathbb{Z}^{k}, c_{t} \circ \phi\right) \rtimes_{\gamma_{t}} \mathbb{Z}^{k},
\end{aligned}
$$

takes $\left[f_{v}\right]$ to $\left[s_{v}\right]$ for any $v \in \operatorname{Obj}(\Lambda)$ and any $t \in[0,1]$. Consequently, the isomorphism $K_{*}\left(C^{*}\left(\Lambda, c_{t}\right)\right) \cong K_{*}\left(C^{*}\left(\Lambda, c_{s}\right)\right)$ preserves the class of $s_{v}$, as claimed.

Remark 4.6. It's tempting to think that, since $C^{*}\left(\Lambda \times_{d} \mathbb{Z}^{k}, c \circ \phi\right) \cong C^{*}\left(\Lambda \times_{d} \mathbb{Z}^{k}\right)$ and $C^{*}(\Lambda, c) \sim_{M E} C^{*}\left(\Lambda \times_{d} \mathbb{Z}^{k}, c \circ \phi\right) \rtimes_{l t} \mathbb{Z}^{k}$ for any cocycle $c$ on $\Lambda$, any two twisted $k$-graph $C^{*}$-algebras should be Morita equivalent. This statement is false, however (the rotation algebras provide a counterexample). The flaw lies in the fact that the isomorphism $\operatorname{Ad} U_{*}: C^{*}\left(\Lambda \times_{d} \mathbb{Z}^{k}, c \circ \phi\right) \rightarrow C^{*}\left(\Lambda \times_{d} \mathbb{Z}^{k}\right)$ is not equivariant 
with respect to the left-translation action of $\mathbb{Z}^{k}$, so the isomorphism

$$
C^{*}\left(\Lambda \times_{d} \mathbb{Z}^{k}, c \circ \phi\right) \cong C^{*}\left(\Lambda \times_{d} \mathbb{Z}^{k}\right)
$$

does not pass to an isomorphism $C^{*}\left(\Lambda \times_{d} \mathbb{Z}^{k}, c \circ \phi\right) \rtimes_{l t} \mathbb{Z}^{k} \rightarrow C^{*}\left(\Lambda \times_{d} \mathbb{Z}^{k}\right) \rtimes_{l t} \mathbb{Z}^{k}$. In other words, a $K$-theoretic equivalence of twisted $k$-graph $C^{*}$-algebras is the best result we can hope for in general.

\section{Future work}

The standing hypotheses of this paper, that our $k$-graphs be row-finite and sourcefree, are slightly more restrictive than the current standard for $k$-graphs. Thus, we would like to extend Theorem 4.1 to apply to all finitely aligned $k$-graphs. Finitely aligned $k$-graphs were introduced in [Raeburn and Sims 2005; Raeburn et al. 2004], and it seems that they constitute the largest class of $k$-graphs to which one can profitably associate a $C^{*}$-algebra. However, the Kumjian-Pask construction of a groupoid $\mathcal{G}_{\Lambda}$ associated to a $k$-graph $\Lambda$, which we described in Section 2 and which we use throughout the proof of Theorem 4.1, only works when $\Lambda$ is row-finite and source-free. Farthing, Muhly, and Yeend [Farthing et al. 2005] provide an alternate construction of a groupoid $\mathcal{G}$ which can be associated to an arbitrary finitely aligned $k$-graph, and we hope that this approach will allow us to apply groupoid results such as Theorem 3.3 to study the effect on $K$-theory of homotopies of cocycles for finitely aligned $k$-graphs.

\section{Acknowledgements}

This research constitutes part of my $\mathrm{PhD}$ thesis, and I would like to thank my advisor, Erik van Erp, for his encouragement and assistance. I would also especially like to thank Prof. Jody Trout for his help and support throughout my PhD work.

\section{References}

[Brown and an Huef 2014] J. H. Brown and A. an Huef, "Decomposing the $C^{*}$-algebras of groupoid extensions", Proc. Amer. Math. Soc. 142:4 (2014), 1261-1274. MR 3162248 Zbl 1302.46053

[Clark and an Huef 2012] L. O. Clark and A. an Huef, "The representation theory of $C^{*}$-algebras associated to groupoids", Math. Proc. Cambridge Philos. Soc. 153:1 (2012), 167-191. MR 3043775 Zbl 1270.46047

[Echterhoff et al. 2010] S. Echterhoff, W. Lück, N. C. Phillips, and S. Walters, "The structure of crossed products of irrational rotation algebras by finite subgroups of $\mathrm{SL}_{2}(\mathbb{Z})$ ", J. Reine Angew. Math. 639 (2010), 173-221. MR 2011c:46127 Zbl 1202.46081

[Farthing et al. 2005] C. Farthing, P. S. Muhly, and T. Yeend, "Higher-rank graph $C^{*}$-algebras: An inverse semigroup and groupoid approach", Semigroup Forum 71:2 (2005), 159-187. MR 2006h:46052 Zbl 1099.46036

[Gillaspy 2015] E. Gillaspy, “ $K$-theory and homotopies of 2-cocycles on transformation groups”, $J$. Operator Theory 73:2 (2015), 465-490. MR 3346133 Zbl 06465653 
[Goehle 2009] G. Goehle, Groupoid crossed products, Ph.D. thesis, Dartmouth College, 2009, available at http://search.proquest.com/docview/230889835.

[an Huef et al. 2011] A. an Huef, A. Kumjian, and A. Sims, "A Dixmier-Douady theorem for Fell algebras”, J. Funct. Anal. 260:5 (2011), 1543-1581. MR 2012i:46066 Zbl 1251.46028

[Kumjian 1986] A. Kumjian, "On $C^{*}$-diagonals", Canad. J. Math. 38:4 (1986), 969-1008. MR 88a: $46060 \mathrm{Zbl} 0627.46071$

[Kumjian and Pask 2000] A. Kumjian and D. Pask, "Higher rank graph $C^{*}$-algebras", New York J. Math. 6 (2000), 1-20. MR 2001b:46102 Zbl 0946.46044

[Kumjian et al. 2013] A. Kumjian, D. Pask, and A. Sims, "On the $K$-theory of twisted higher-rankgraph $C^{*}$-algebras”, J. Math. Anal. Appl. 401:1 (2013), 104-113. MR 3011251 Zbl 1279.46053

[Kumjian et al. 2015] A. Kumjian, D. Pask, and A. Sims, "On twisted higher-rank graph $C^{*}$-algebras", Trans. Amer. Math. Soc. 367:7 (2015), 5177-5216. MR 3335414 Zbl 06429159

[Muhly and Williams 1992] P. S. Muhly and D. P. Williams, "Continuous trace groupoid $C^{*}$-algebras, II”, Math. Scand. 70:1 (1992), 127-145. MR 93i:46117 Zbl 0783.46028

[Muhly et al. 1996] P. S. Muhly, J. N. Renault, and D. P. Williams, "Continuous-trace groupoid $C^{*}$ algebras, III”, Trans. Amer. Math. Soc. 348:9 (1996), 3621-3641. MR 96m:46125 Zbl 0859.46039

[Pimsner and Voiculescu 1980] M. Pimsner and D. Voiculescu, "Exact sequences for $K$-groups and Ext-groups of certain cross-product $C^{*}$-algebras", J. Operator Theory 4:1 (1980), 93-118. MR 82c:46074 Zbl 0474.46059

[Raeburn and Sims 2005] I. Raeburn and A. Sims, "Product systems of graphs and the Toeplitz algebras of higher-rank graphs", J. Operator Theory 53:2 (2005), 399-429. MR 2006d:46073 Zbl 1093.46032

[Raeburn et al. 2004] I. Raeburn, A. Sims, and T. Yeend, "The $C^{*}$-algebras of finitely aligned higher-rank graphs”, J. Funct. Anal. 213:1 (2004), 206-240. MR 2005e:46103 Zbl 1063.46041

[Renault 1980] J. Renault, A groupoid approach to $C^{*}$-algebras, Lecture Notes in Mathematics 793, Springer, Berlin, 1980. MR 82h:46075 Zbl 0433.46049

[Robertson and Steger 1999] G. Robertson and T. Steger, "Affine buildings, tiling systems and higher rank Cuntz-Krieger algebras", J. Reine Angew. Math. 513 (1999), 115-144. MR 2000j:46109 Zbl 1064.46504

[Tu et al. 2004] J.-L. Tu, P. Xu, and C. Laurent-Gengoux, "Twisted $K$-theory of differentiable stacks", Ann. Sci. École Norm. Sup. (4) 37:6 (2004), 841-910. MR 2005k:58037 Zbl 1069.19006

[Williams 2007] D. P. Williams, Crossed products of $C^{*}$-algebras, Mathematical Surveys and Monographs 134, Amer. Math. Soc., Providence, RI, 2007. MR 2007m:46003 Zbl 1119.46002

Received April 2, 2014. Revised March 25, 2015.

\section{ELIZABETH GILLASPY}

DEPARTMENT OF MATHEMATICS

UNIVERSITY OF COLORADO - BOULDER

CAMPUS BoX 395

BOULDER, CO 80309-0395

UNITED STATES

elizabeth.gillaspy@colorado.edu 


\title{
PACIFIC JOURNAL OF MATHEMATICS
}

\author{
msp.org/pjm
}

Founded in 1951 by E. F. Beckenbach (1906-1982) and F. Wolf (1904-1989)

\section{EDITORS}

Don Blasius (Managing Editor)

Department of Mathematics

University of California

Los Angeles, CA 90095-1555

blasius@math.ucla.edu

\author{
Paul Balmer \\ Department of Mathematics \\ University of California \\ Los Angeles, CA 90095-1555 \\ balmer@math.ucla.edu \\ Robert Finn \\ Department of Mathematics \\ Stanford University \\ Stanford, CA 94305-2125 \\ finn@math.stanford.edu \\ Sorin Popa \\ Department of Mathematics \\ University of California \\ Los Angeles, CA 90095-1555 \\ popa@math.ucla.edu
}

\author{
Vyjayanthi Chari \\ Department of Mathematics \\ University of California \\ Riverside, CA 92521-0135 \\ chari@math.ucr.edu \\ Kefeng Liu \\ Department of Mathematics \\ University of California \\ Los Angeles, CA 90095-1555 \\ liu@math.ucla.edu \\ Jie Qing \\ Department of Mathematics \\ University of California \\ Santa Cruz, CA 95064 \\ qing@ cats.ucsc.edu
}

\section{PRODUCTION}

Silvio Levy, Scientific Editor, production@msp.org

\section{SUPPORTING INSTITUTIONS}

ACADEMIA SINICA, TAIPEI

CALIFORNIA INST. OF TECHNOLOGY

INST. DE MATEMÁTICA PURA E APLICADA

KEIO UNIVERSITY

MATH. SCIENCES RESEARCH INSTITUTE

NEW MEXICO STATE UNIV.

OREGON STATE UNIV.

\author{
STANFORD UNIVERSITY \\ UNIV. OF BRITISH COLUMBIA \\ UNIV. OF CALIFORNIA, BERKELEY \\ UNIV. OF CALIFORNIA, DAVIS \\ UNIV. OF CALIFORNIA, LOS ANGELES \\ UNIV. OF CALIFORNIA, RIVERSIDE \\ UNIV. OF CALIFORNIA, SAN DIEGO \\ UNIV. OF CALIF., SANTA BARBARA
}

\author{
Daryl Cooper \\ Department of Mathematics \\ University of California \\ Santa Barbara, CA 93106-3080 \\ cooper@math.ucsb.edu \\ Jiang-Hua Lu \\ Department of Mathematics \\ The University of Hong Kong \\ Pokfulam Rd., Hong Kong \\ jhlu@maths.hku.hk \\ Paul Yang \\ Department of Mathematics \\ Princeton University \\ Princeton NJ 08544-1000 \\ yang@math.princeton.edu
}

These supporting institutions contribute to the cost of publication of this Journal, but they are not owners or publishers and have no responsibility for its contents or policies.

See inside back cover or msp.org/pjm for submission instructions.

The subscription price for 2015 is US \$420/year for the electronic version, and \$570/year for print and electronic.

Subscriptions, requests for back issues and changes of subscribers address should be sent to Pacific Journal of Mathematics, P.O. Box 4163, Berkeley, CA 94704-0163, U.S.A. The Pacific Journal of Mathematics is indexed by Mathematical Reviews, Zentralblatt MATH, PASCAL CNRS Index, Referativnyi Zhurnal, Current Mathematical Publications and Web of Knowledge (Science Citation Index).

The Pacific Journal of Mathematics (ISSN 0030-8730) at the University of California, c/o Department of Mathematics, 798 Evans Hall \#3840, Berkeley, CA 94720-3840, is published twelve times a year. Periodical rate postage paid at Berkeley, CA 94704, and additional mailing offices. POSTMASTER: send address changes to Pacific Journal of Mathematics, P.O. Box 4163, Berkeley, CA 94704-0163.

PJM peer review and production are managed by EditFLOW ${ }^{\circledR}$ from Mathematical Sciences Publishers.

\section{PUBLISHED BY}

\section{mathematical sciences publishers \\ nonprofit scientific publishing}

http://msp.org/

(C) 2015 Mathematical Sciences Publishers 


\section{PACIFIC JOURNAL OF MATHEMATICS}

Volume $278 \quad$ No. $2 \quad$ December 2015

Differential Harnack and logarithmic Sobolev inequalities along

Ricci-harmonic map flow

ABIMBOLA ABOLARINWA

On $J$-holomorphic curves in almost complex manifolds with

291

asymptotically cylindrical ends

ERKAO BAO

Integration of coupling Dirac structures

OLIVIER BRAHIC and RUI LOJA FERNANDES

Asymptotic behavior of Palais-Smale sequences associated with fractional Yamabe-type equations

Yi FANG and MARÍA DEL MAR GONZÁLEZ

$K$-theory and homotopies of 2-cocycles on higher-rank graphs

ELIZABETH GILLASPY

Fusion products and toroidal algebras

DENIZ KUS and PETER LiTTELMANN

Differential Harnack estimates for positive solutions to heat equation under Finsler-Ricci flow

\section{SAJJAD LAKZIAN}

On the one-endedness of graphs of groups

NichOLAS TOUIKAN

On the structure of vertex cuts separating the ends of a graph

GARETH R. WILKES 